$\begin{array}{cl}\begin{array}{c}\text { Revue } \\ \text { de /histoire }\end{array} & \text { Revue de l'histoire des religions } \\ \text { des religions } & \begin{array}{l}3 \mid 2013 \\ \text { Varia }\end{array}\end{array}$

\title{
Le jansénisme des Dames Maltaises. Genre, savoir théologique et publicité (Toulouse, 1659-1661) [I]
}

The Dames Maltaises and their Jansenism. Gender, Theological Knowledge and Publicity in Seventeenth Century Toulouse (1659-1661) [I]

Jean-Pascal Gay

\section{CpenEdition}

\section{Journals}

Édition électronique

URL : http://journals.openedition.org/rhr/8123

DOI : 10.4000/rhr.8123

ISSN : 2105-2573

Éditeur

Armand Colin

Édition imprimée

Date de publication : 1 septembre 2013

Pagination : 355-383

ISBN : $978-2200928650$

ISSN : 0035-1423

\section{Référence électronique}

Jean-Pascal Gay, «Le jansénisme des Dames Maltaises. Genre, savoir théologique et publicité (Toulouse, 1659-1661) [1] », Revue de l'histoire des religions [En ligne], 3 | 2013, mis en ligne le 01 septembre 2016, consulté le 01 mai 2019. URL : http://journals.openedition.org/rhr/8123 ; DOI $10.4000 /$ rhr.8123 


\section{Le jansénisme des Dames Maltaises Genre, savoir théologique et publicité (Toulouse, 1659-1661) [I]}

Cet article propose de revenir sur l'appréhension historique du jansénisme du XVII siècle à travers le prisme du genre. Il le fait en étudiant le cas peu connu des Dames Maltaises de Toulouse. Dans un premier temps (I), on analyse la manière par laquelle la question du jansénisme des sœurs émerge sur la scène toulousaine, et les difficultés pour caractériser leur hétérodoxie entre théologie et mystique. Dans un second temps (II), on examine la manière dont les acteurs de ce qui apparaît comme une affaire recourent aux catégories de genre et comment l'usage de ces catégories est aussi lié à des manières de construire la publicité de l'épisode par l'écrit. Dès lors si l'on peut discerner un féminisme chez les Maltaises de Toulouse, il apparaît immédiatement complexe et lié à une configuration sociale et politique précise.

\section{The Dames Maltaises and their Jansenism. Gender, Theological Knowledge and Publicity in Seventeenth Century Toulouse (1659-1661) [I]}

This paper discusses the historical apprehension of early Jansenism through gender. It investigates the somewhat forgotten case of the Dames Maltaises in Toulouse. A first instalment (I) shall explore the emergence of the issue of the nuns' Jansenism on the local scene of Toulouse as well as the difficult characterization of their heterodoxy between theology and mysticism. A second instalment (II) shall analyse the uses of gender categories in this affair and their connection to the way this episode was publicised through writing. If some form of feminism can be identified here, it immediately appears as complex and tied to a specific social and political context. 
La branche féminine de l'ordre de Malte, les sœurs Hospitalières de Saint-Jean-de-Jérusalem - les «Dames Maltaises» - joue un rôle très significatif dans la vie religieuse des noblesses féminines en particulier dans l'Europe méditerranéenne'. La communauté moderne de Toulouse est connue des historiens du jansénisme en raison de sa mention par Godrefroy Hermant qui consacre le $29^{\mathrm{e}}$ chapitre de son $24^{\mathrm{e}}$ livre, relatant les événements du début de la première moitié de l'année 1661, aux «Religieuses maltoises de Toulouse persécutées pour le jansénisme», affaire qui «n'eut pas de longues suites, ces religieuses ne s'étant pas trouvées à l'épreuve d'une grande persécution $»^{2}$. Dans l'économie générale des Mémoires, le chapitre ne trouve guère sa place, sinon par les pièces qu'il suscite à Paris. La disproportion entre l'importance de ce qui, à Toulouse, est bien une «affaire», et son appréciation a minima par le mémorialiste interroge doublement. Elle signale, d'une part, la complexité des rapports entre les différentes scènes et les différents publics des controverses jansénistes; et, d'autre part, la diversité des destins religieux, politiques, mais aussi historiques et mémoriels des jansénismes féminins au $\mathrm{XVII}^{\mathrm{e}}$ siècle. Alors que la mise en scène de la résistance du monastère de Port-Royal est au cœur de l'écriture du mouvement et de la construction d'un habitus janséniste, alors qu' une autre affaire toulousaine - celle des Filles de l'Enfance - joue un rôle significatif dans la construction de la mémoire janséniste et les transformations du jansénisme à partir de cette élaboration mémorielle ${ }^{3}$ entre $\mathrm{XVII}^{\mathrm{e}}$ et $\mathrm{XVIII}^{\mathrm{e}}$ siècles,

1. Pour un aperçu de l'histoire de la congrégation des Dames Maltaises, voir Joseph Delaville Le Roulx, «Les Hospitalières de Saint-Jean-de-Jérusalem», Compte-rendus des séances de l'Académie des Inscriptions et Belles-Lettres, 38/2 (1894), p. 137-146; à propos de leur présence dans le Sud-Ouest français, Edmond Albe «Les religieuses hospitalières de l'Ordre de Saint-Jean-de-Jérusalem au diocèse de Cahors», Revue d'histoire de l'Église de France, 27, 112 (1941), p. $180-220$.

2. Godefroy Hermant, Mémoires sur l'histoire ecclésiastique du XVII siècle (1630-1663), Augustin Gazier (éd.), vol. Iv, Paris, 1905-1910, p. 700-707.

3. Sur le rôle de la mémoire dans la transformation pratique du jansénisme, voir Catherine Maire, De la cause de Dieu à la cause de la Nation, Paris, Gallimard, 1998, p. 44-45. S'agissant des Filles de l'Enfance, Arnauld en proposa une défense passionnée, qui lie le sort de la congrégation toulousaine à celui du monastère des Champs, dans L'Innocence opprimée par la calomnie ou l'histoire 
la «persécution» des Dames Maltaises n'y a, elle, guère laissé de traces. Cette disproportion explique certainement le peu de travaux qu'a suscité cet épisode en dehors de ceux de l'historien (antijanséniste) du jansénisme toulousain, Alphonse Auguste ${ }^{4}$.

Or, un des lieux récents de renouvellement des études sur le premier jansénisme a été l'attention portée aux engagements féminins dans la déviance religieuse et politique qu'il a étés. Daniella Kostroun insiste en particulier sur le caractère proprement féministe - à condition d'en accepter une définition paradoxale et spécifique à l'époque moderne - de la résistance du monastère de Port-Royal. Les sœurs, en recourant au langage des droits universels pour revendiquer une véritable égalité civique sinon religieuse et en mobilisant des modèles féminins d'autorité, réussissent à placer la monarchie devant les contradictions de ses exigences. On ajoutera qu'en retour, la spécificité de cette contribution féminine ouvre un espace d'écriture pour l'illustration plus générale de ces contradictions, dans un espace public qui n'est pas seulement féminin. Le cas de l'accusation de jansénisme contre le monastère toulousain

de la Congrégation des Filles de l'Enfance. L'épisode est régulièrement cité au milieu du XVIII ${ }^{e}$ siècle. Il est ainsi mentionné, à charge contre le P. de La Chaize dans l'Histoire générale de la naissance et des progrès de la Compagnie de Jésus et Analyse de ses Constitutions et privilèges [p. 116]. Pour l'histoire de la congrégation, voir les travaux de Marguerite-Marie Shibano, infra, note 6.

4. Il consacre un article spécifique à l'affaire des Dames Maltaises, «PortRoyal à Toulouse, ou le jansénisme des religieuses maltaises sous l'épiscopat de P. de Marca (1652-1662)», Revue historique de Toulouse, t. 7, 1920, p. 161-189. L'auteur a eu accès à certaines pièces que nous n'avons pas retrouvées mais pas au dossier conservé aux Archives de la Congrégation pour la Doctrine de la Foi qui permet d'y voir plus clair sur l'ensemble de l'affaire.

5. Fr. Ellen Weaver, Madame de Fontpertuis. Une dévote janséniste, amie et gérante d'Antoine Arnauld et de Port-Royal, Paris, Klincksieck, 1998, et Mademoiselle de Joncoux. Polémique janséniste à la veille de la bulle Unigenitus, Paris, Cerf, 2002; «Erudition, Spirituality and Women: The Jansenist Contribution», dans Sh. Marshall (éd.), Women in Reformation and CounterReformation Europe: Public and Private Worlds, Indianapolis, Indiana University Press, 1989, p. 189-206. Et de Daniella Kostroun, «A Formula for Disobedience: Jansenism, Gender, and the Feminist Paradox », Journal of Modern History, 75/3 (2003), p. 483-552 ainsi que Feminism, Absolutism and Jansenism. Louis XIV and the Port-Royal Nuns, Cambridge, Cambridge University Press, 2011. Pour une reprise critique récente pour le XVIII" siècle, voir Nicolas Lyon-Caen " "Il faut qu'un party se sente bien faible quand il accepte et recherche de tels appuis". Femmes, jansénisme et publicité à Paris au XVIII ${ }^{e}$ siècle » dans L'Atelier du Centre de Recherches Historiques, 4, 2009, [en ligne], mis en ligne le 26 juillet 2009. URL: http//acrh.revues.org/index 1277.html. 
de la branche féminine d'un ordre militaire signale cependant que les engagements féminins n'offrent pas tous les mêmes ressources pour le mouvement dans son ensemble, ce qui en retour peut affecter les formes prises par cet engagement. Réintroduire alors la publicité et le devenir d'engagement féminins relativement publics car ils sont, malgré la clôture, portés en corps par des communautés bien insérées dans des réseaux politiques, sociaux et religieux, conduit-il à envisager différemment l'interaction entre genre et jansénisme?

De ce point de vue, l'épisode du jansénisme des Dames Maltaises est particulièrement significatif. L'affaire émerge en effet dans les relations internes à l'ordre et dans les conflits toulousains au milieu de l'année 1659, gagne en importance et en publicité du fait de la présence de la Cour dans la ville au moment de la Paix des Pyrénées à la fin de l'année, avant de se déployer dans une séquence de publications polémiques en 1661, puis de se résoudre dans un retour au silence dès la fin de l'année. On se propose donc de revenir sur cet échec de la publicisation pour interroger la manière dont l'analyse des régimes de publicité des jansénismes féminins permet d'en éclairer la nature et les mécanismes.

\section{L'invention du Jansénisme des Dames Maltaises SUR LA SCÈNE TOULOUSAINE}

Le monastère des Dames Maltaises est, au moment de cet épisode, de fondation assez récente. Les sœurs, une des quatorze communautés féminines implantées dans l'espace urbain toulousain au XVII ${ }^{\mathrm{e}}$ siècle, se sont en effet installées au faubourg Saint-Cyprien en $1624^{6}$. La ville est un espace d'essor du premier jansénisme marqué par le conflit doctrinal ${ }^{7}$. Plusieurs familles

6. Philippe Wolff, Le Diocèse de Toulouse, Histoire des Diocèses de France, $\mathrm{n}^{\circ}$ 15, Paris, Beauchesne 1983, p. 140.

7. Sur le premier jansénisme toulousain, on trouvera un résumé utile dans Robert A. Schneider, Public Life in Toulouse, 1463-1789. From Municipal Republic to Cosmopolitan City, Ithaca, Cornell University Press, 1989, p. 212219. Voir aussi le résolument antijanséniste Alphonse Auguste, Les origines du Jansénisme dans le diocèse de Toulouse, Paris, Picard, 1922, ainsi que les travaux non publiés de Marguerite-Marie Shibano, Les Débuts de la Congrégation des Filles de l'Enfance de N.-S.-J.-C.: les fondateurs: Gabriel de Ciron et Mme de Mondonville, mémoire dactylographié, Paris, EPHE, 1977 et De la fondation 
de magistrats ont des liens personnels avec Port-Royal et avec les évêques pyrénéens favorables au jansénisme. La Compagnie du Saint-Sacrement dans les années 1650 y semble dominée par ses partisans; cette orientation philo-janséniste de sa branche toulousaine a pu être une des raisons de la fondation d'une $A A$ concurrente $^{8}$. De même, la société féminine des Dames du SaintSacrement, parmi lesquelles Madame de Mondonville9 convertie en 1653 par l'abbé de Ciron ${ }^{10}$ qui semble jouer un rôle essentiel dans les milieux parlementaires. Sous l'épiscopat de Charles de Montchal, approbateur de la Fréquente Communion (1643) et proche de l'évêque de Pamiers, Caulet, Gabriel de Ciron avait participé à la fondation du séminaire des Hautes Études, dont la direction est assurée par le P. Vignaux, clerc au cœur de l'affaire des Dames Maltaises; le séminaire est fermé l'année même de la crise et Madame de Mondonville fondera trois ans plus tard la congrégation des Filles de l'Enfance, dont l'histoire constitue une étape cruciale de la reprise de la répression du jansénisme après la rupture de la paix de l'Église, mais aussi des transformations du rapport entre mémoire et écriture si caractéristiques du jansénisme de la fin du $\mathrm{XVII}^{\mathrm{e}}$ siècle $^{11}$. L'opposition entre jansénisme et anti-jansénisme recoupe et renouvelle par ailleurs certainement l'opposition entre les pénitents noirs et bleus ${ }^{12}$.

Compte tenu d'une part de l'accueil fait à l'augustinisme dans la ville et notamment dans sa noblesse, et d'autre part de la présence de Vignaux dans l'entourage du monastère, il n'est pas surprenant que les sœurs du faubourg Saint-Cyprien aient déjà fait assez tôt l'objet de soupçons, comme l'atteste une lettre du Grand-Maître

de la Congrégation des Filles de l'Enfance à la deuxième persécution, mémoire dactylographié, Paris, EPHE, 1980.

8. Henri Bégouen, Une société secrète émule de la Compagnie du SaintSacrement: l'AA de Toulouse aux XVII et XVIII siècles, Toulouse, Privat, Paris, Picard, 1913; R. A. Schneider, op. cit., p. 214.

9. Dictionnaire de Port-Royal, Paris, Honoré Champion, 2004, col. 740.

10. «Gabriel de Ciron», Dictionnaire de Port-Royal, p. 274-275. M.-M. Shibano «Gabriel de Ciron (1619-1673), Esquisse biographique», Revue d'Histoire de la spiritualité, 52, 1976, p. 89-124.

11. Sur le jansénisme déclaré du P. Vignaux, A. Auguste, Les origines du Jansénisme dans le diocèse de Toulouse, op. cit., p. 87; et Le Séminaire de Caraman au Faubourg Saint-Étienne à Toulouse, Paris, Picard, 1913, p. 1, 15, 36-43, 157-158; Clément Tournier, Histoire des séminaires toulousains, Toulouse, Imprimerie Fournié, 1942, p. 16-19; R. A. Schneider, op. cit., p. 216.

12. R. A. Schneider, op. cit., p. 216. 
Paul de Vintimille Lascaris aux sœurs, évoquant des rumeurs selon lesquelles «il y a danger que parmy vous, il n'y ait des esprits déjà gâtés par le livre du docteur Arnault». À ce stade cependant, il ne semble pas qu'aucune décision d'examen ait été prise. Par ailleurs, l'origine des rumeurs est incertaine. Par la suite, plusieurs sœurs en accuseront les jésuites. Il semble cependant que la demande d'une visite canonique du monastère soit issue de quelques-unes des sœurs insatisfaites de l'évolution et du gouvernement du monastère ${ }^{13}$. La question des liens avec la Compagnie est cependant bien présente; elle révèle non pas tant l'ethos de chacune des différentes parties prenantes des conflits qui divisent alors le catholicisme français, que la capacité de ces divisions à peser sur la vie des communautés régulières par les pratiques qu'elles acculturent.

Quoi qu'il en soit, le successeur de Lascaris à la tête de l'Ordre de Malte, Martin de Redin ${ }^{14}$, commet le 3 avril 1659 les commandeurs Jacques de Carbonneau et Jacques de Lancègue pour la visite du couvent. L'acte de commission, qui passe par la chancellerie de l'ordre, est accompagné d'un «écrit secret » ${ }^{15}$, précisant son contenu réel. Tout en refusant de croire que le jansénisme se soit répandu dans le couvent toulousain, le Grand-Maître affirme la nécessité « d'agir pour son remède comme si nous le croions ». La visite devra donc commencer par la remise aux commissaires de l'ensemble des «livres imprimez ou manuscrits, [...], et généralement toutes les escritures qu'elles auront en leur pouvoir [...]». Les commissaires d'ailleurs ne doivent pas conserver les ouvrages par-devers eux mais les consigner «à deux religieux des ordres suivants, sçavoir, Dominiquains, Franciscains, Minimes, Feuillans, Jésuistes, Carmes et Bénédictins », leur censure, jointes aux «informations secrètes» des commissaires, devant permettre d'établir la vérité de la cause. En cas de présence du jansénisme dans la maison, les commissaires doivent faire cesser tous contacts avec les religieux de l'Oratoire et de la Mission, sauf permission du Grand-Maître, et interdire l'accès

13. Voir lettre du 8 juillet 1659 au Grand-Maître, Archivio della Congregazione per la Dottrina della Fede (Cité du Vatican), désormais abrégé ACDF, St. St. F2a, 570, dans laquelle est mentionnée une demande de nommer des visiteurs (voir infra).

14. Voir la liste et la chronologie des Grands-Maîtres ayant eu à traiter l'affaire des religieuses toulousaines dans, A. Auguste, art. cit., p. 165, n. 3. (Lascaris est Grand-Maître de 1636 à 1657, Redin de 1657 à 1660, Cotoner de 1660 à 1663.)

15. ACDF, St. St. F 2a, 542. 
du couvent aux personnes, de «quelle condition qu'elle[s] soi[en]t, qui soi[en]t soubçonnée[s] d'adhérer à ses [sic] opinions ».

La visite commence le 23 mai $1659^{16}$ : les commissaires imposent aux sœurs de s'ouvrir en conscience à eux et de ne plus recevoir au parloir le temps de la visite. Ils récoltent les écrits et en font des ballots individuels. Les livres sont mis dans la chambre du confesseur de la maison et séquestrés. Le lendemain, les livres suspects sont envoyés aux censeurs. Le 29 mai les commissaires visitent l'église et examinent les fondations liées à la chapelle. Alphonse Auguste, dans l'article qu'il consacre à l'épisode, relève qu'un des obits est desservi par Arnaud Baric ${ }^{17}$, une des figures du premier jansénisme toulousain ${ }^{18}$. C'est le même jour qu'une sœur d'office demande à voir les commissaires et se plaint du directeur qui est le sien depuis deux ou trois ans, M. Vignaux, «lequel lui auroit mis dans l'esprit beaucoup de choses touchant son salut, lui parlant de la grâce en façons que cette pauvre fille [leur] dict qu'elle ne trouvoit point de repos et ne sçavoit comme quoy soulager son âme des troubles et angoisses dans lesquelles elle étoit plongée à cause de la conduite que ledit sieur Vignaux lui avoit mise dans la tête». Les commissaires lui recommandent un changement de directeur et font solliciter pour elle, qui demande un jésuite, le P. Jean Ferrier (futur confesseur du Roi), professeur de théologie dans le Collège de Toulouse. Le lendemain, le P. Ferrier se présente au monastère, où la portière le reçoit en s'étonnant «bien fort que les Jésuites allassent là-dedans, attendu qu'ils étoient leurs ennemis et leurs dénonciateurs ». Plusieurs sœurs se joignent à elle pour leur reprocher l'accusation de jansénisme. Ferrier se rend ensuite auprès de Carbonneau qui s'excuse de la conduite des sœurs. Le visiteur fait alors signifier aux moniales qu'il ne veut plus retourner dans leur maison pour terminer la visite «de peur qu'elles ne [le] traittassent comme elles avoient fait le père Ferrier». Les sœurs s'excusent à leur tour auprès du visiteur, qui refuse de les recevoir tant qu'elles

16. Procès-verbal de la visite aux archives départementales de la HauteGaronne 216H 35 [fonds des Dames Maltaises; cité désormais comme Procèsverbal]. A. Auguste, art. cit., s'appuie largement sur ce document dont il ne donne pas la colocation. Le document n'est pas paginé.

17. A. Auguste, art.cit., p. 170.

18. Sur Arnaud Baric, voir M.-M. Shibano, «Arnaud Baric, missionnaire apostolique: un prêtre en guerre contre la pauvreté, la peste et les Jésuites (16071668)», Annales du Midi, 100, 1988, p. 153-180. 
n'auront pas fait amende honorable auprès du P. Ferrier. Ferrier effectue alors une seconde visite, sans parvenir à voir la religieuse concernée. Pour «ne pas gaster l'affaire davantage», il ne pousse cependant pas plus avant et se déclare satisfait au commissaire du Grand-Maître. Ceci ne règle cependant pas le conflit entre le visiteur et la communauté, ce dernier leur reprochant longuement leur désobéissance et refusant de poursuivre la visite. Les choses se sont alors suffisamment envenimées pour devenir l'objet de la rumeur publique.

Le procès-verbal de la visite ne reprend qu'à la date du 3 juillet, avec l'audition des sœurs. Mais elle est assez vite interrompue car celles-ci ont écrit au Grand-Maître pour se plaindre du traitement des commissaires et réclament la suspension de la visite tant qu'elles n'ont pas reçu des ordres nouveaux de sa part. Les visiteurs cèdent à leurs instances, "pour esviter qu'elles ne fissent des actions de désobéissance» mais leur imposent de ne recevoir aucun ecclésiastique sans leur accord ${ }^{19}$. Ils écrivent de leur côté au GrandMaître pour demander effectivement à suspendre leur visite ${ }^{20}$, et transmettent en même temps la censure des ouvrages retrouvés chez les moniales. Car entre juillet et août s'est effectué le tri entre les écrits des sœurs: ceux jugés inoffensifs leur ont été rendus quand les écrits suspects sont demeurés entre les mains des censeurs pour en dresser une censure formelle.

La visite n'a pas été sans effet sur la communauté. Les commissaires, selon le rapport que Carbonneau en fait au GrandMaître $^{21}$, ont eu de longs entretiens privés avec les sœurs. Il indique qu'il «y a eu cinq ou six Religieuses si zélées qu'elles ont dressés en leur particulier des mémoires sur les abus qu'elles ont cru estre nécessaires d'estre corrigés». Le P. Rapin, qui comme Hermant mentionne l'épisode dans ses mémoires, revient en détail sur cette

19. Procès-verbal en date du 9 août. Ordonnance des visiteurs du 9 août 1659 , ACDF, St. St. F 2a, 544. En réalité l'ordonnance n'est pas appliquée puisque le 10, toujours par crainte de la désobéissance des sœurs, ils donnent pouvoir à la prieure d'accorder dispense sur ce point, mais en lui précisant les noms des ecclésiastiques à exclure, de sorte que si la supérieure «en usast autrement ce feust par sa faute et non pas de $\mathrm{n}[\mathrm{ot}] \mathrm{re}$ consentement» (procès-verbal, ibid.).

20. Voir la lettre de Girolamo Casanate, alors Inquisiteur à Malte, à Mgr Vizzani, du 6 octobre 1659 l'informant de cette demande de suspension, ACDF, St. St. F 2a, 543 et la copie non datée de la lettre non datée de demande de suspension de la visite de Carbonneau et Lancègue, ACDF, St. St. F 2a, 566.

21. ACDF, St. St. F 2a, 566. 
division $^{22}$. Trois sœurs, Antoinette et Françoise de Mirandol, ainsi que Perside de Bénac de Navailles, auraient écrit au Grand-Maître pour lui demander la reprise de la visite. Il faut y ajouter, comme l'indique la copie des archives de l'Inquisition, une quatrième signataire, F. de Fontenilles. Leur lettre datée du 8 juillet 1659 et dont une copie est préservée dans les archives de l'Inquisition ${ }^{23}$ n'infirme pas Rapin, qui semble relativement bien informé. Outre qu'elle dénonce «les mauvaises maximes et doctrines qui depuis quelques années [se] sont glissées » dans le monastère, elle s'attarde sur la dérive partisane que ses auteures attribuent à «trois sœurs [...] qui sont absolues céans et qui font des officières à leur poste et de leur cabale». La lettre insiste par ailleurs sur l'adversité à l'égard de la Compagnie de Jésus qui anime le parti dominant, sous l'influence des Oratoriens, mais encore des pères de la Mission, des Carmes déchaux et des Doctrinaires «tous lesquels ensemble ont donné de grandes aversions contre les Révérends Pères Jésuites» parce que, précisent les sœurs, «ils sont ennemis de ces nouvelles doctrines». Ce groupe est par ailleurs clairement appuyé par les visiteurs puisque ceux-ci suggèrent au Grand-Maître de rendre à «Madame de Mirandol, prieure ancienne» le priorat. Ils avancent cet expédient comme «l'unique remède, le plus doux et le plus prudent de remettre cette maison». Par contraste implicite, la visite apparaît alors en creux, du moins à ce stade, comme une solution violente et inadéquate, même à ceux qui sont chargés de la mettre en œuvre.

\section{L'IMPOSSIBLE CLÔTURE D’UN CONFLIT PUBLIC}

Ce n'est cependant pas la solution proposée par les visiteurs qui est finalement adoptée par le Grand-Maître. En réalité ce dernier agit alors essentiellement sur ordre de Rome et de l'Inquisition. La première lettre dont on dispose date du 29 juillet $1659^{24}$ mais signale que Rome s'emploie déjà à s'informer de ce qui se passe dans le monastère toulousain. À ce moment, l'Inquisiteur de Malte, Girolamo Casanate, demande à ce qu'on insiste auprès 
du Grand-Maître pour que la visite continue, en impliquant désormais l'archevêque. Le Grand-Maître semble d'ailleurs aller dans son sens. Il transmet plusieurs pièces venues de Toulouse à l'Inquisiteur que ce dernier peut alors faire parvenir à Rome pour obtenir des ordres plus précis. C'est sur la base de ces écrits que le consulteur Lorenzo di Lauria, qui joue déjà un rôle important dans les congrégations du jansénisme ${ }^{25}$, approuve la censure théologique faite par les examinateurs toulousains ${ }^{26}$. L'essentiel de son votum porte sur la conduite à tenir à l'égard des moniales. C'est lui qui recommande de ne pas procéder juridiquement contre les sœurs, mais de s'employer à rétablir la paix dans le monastère, par des confesseurs extraordinaires, et neutres théologiquement, chargés de détromper les sœurs de leurs erreurs et surtout de s'employer à rétablir une saine dévotion. Ce sont les instructions qui sont transmises à l'archevêque.

Ce dernier, Pierre de Marca, est poussé à l'action non seulement par le Grand-Maître et le nonce mais aussi par le roi et la reinemère ${ }^{27}$. La famille royale est en effet arrivée à Toulouse le 14 octobre. Le voyage intervient alors qu'est signé à Saint-Jean-de-Luz, le 7 novembre 1759, le traité des Pyrénées. C'est aussi l'occasion d'une réunion des États de Languedoc. Le séjour de la Cour, accompagnée du nonce, dure jusqu'au 28 décembre, la famille royale logeant au palais archiépiscopal ${ }^{28}$. Comme de coutume dans ce type de circonstances, le roi honore de sa présence plusieurs communautés religieuses, marquant cependant une certaine prédilection pour les jésuites en se rendant dans l'église de la maison professe deux fois, notamment à l'occasion de la fête de saint François-Xavier le 2 décembre. Le lendemain il assiste à une représentation d'une

25. Sur son rôle dans les débats romains autour et dans la suite des Provinciales, voir Jean-Louis Quantin, «Si mes lettres sont condamnées à Rome. Les Provinciales devant le Saint-Office», à paraître dans Dominique Descotes (éd.), Pascal et les Jésuites. Le retentissement des Provinciales. Une première version ramassée de ce texte est parue dans Courrier du Centre international Blaise Pascal, 29 (2007), p. 10-25; je remercie l'auteur de m'avoir donné accès à la version la plus étoffée de ce texte important.

26. «Iudicium theologorum, quo qualificant praefata scripta est sanum, et prudens».

27. Voir la lettre de Marca au Grand-Maître du 24 janvier 1660, ACDF, St. St. F 2a, 577.

28. Christophe Levantal, Louis XIV. Chronographie d'un règne, Gollion, Infolio, 2009, p. 182-183. 
pièce jouée par les écoliers du collège en célébration de la Paix ${ }^{29}$. Faut-il lier cette prédilection affichée au contexte de développement d'un premier jansénisme ? Rien ne permet de le dire. Reste qu'à une date indéterminée, mais cependant certainement avant la reprise de la visite canonique le 23 octobre $^{30}$, Louis XIV et Anne d'Autriche visitent le monastère des Dames Maltaises pour y entendre la messe ${ }^{31}$. Tous deux se déclarent satisfaits et impressionnés aux dires de Marca «par leur modestie religieuse, et la contenance sérieuse qui marquoit leur bonne naissance et leur éducation». La reine aurait même témoigné son désir «de faire un semblable établissement à Paris; les filles de bonne noblesse auroient plus de satisfaction de passer leur vie en servant Dieu dans une communauté composée de personnes de condition». C'est alors cependant que la Cour a vent des doutes qui pèsent sur l'orthodoxie des sœurs, signe probable de la faible publicité de l'affaire à ce stade, la visite intervenant au début du séjour toulousain du souverain. La reine informée ensuite du soupçon de jansénisme pesant sur la communauté affirme publiquement regretter une visite qu'elle n'aurait pas faite si elle en avait eu connaissance. Elle ordonne alors à Marca de prendre en main la visite comme le Grand-Maître Redin l'en a sollicités2.

La visite reprend donc fin octobre. Redin demande aux commissaires de soumettre le choix des prédicateurs, directeurs et confesseurs des sœurs à l'archevêque. Celles-ci y trouvent un argument canonique pour retarder encore la visite. Elles se plaignent qu'une telle disposition est contraire à leurs règles, opposant dans un geste comparable à celui des sœurs de Port-Royal leur droit naturel et particulier à des exigences d'obéissance exorbitantes ${ }^{33}$.

29. Ibid. L'auteur s'appuie ici sur la Gazette. Il indique que la représentation du Siècle d'Or captif mis en liberté par la Paix a été donnée par les écoliers de la maison-professe. Il s'agit bien sûr de ceux du collège (voir Robert Mesuret, Le Théâtre à Toulouse de 1561 à 1914, Toulouse, musée Paul-Dupuy, 1972, p. 16).

30. La visite interrompue depuis le 9 août reprend à cette date, moment où Carbonneau signifie aux sœurs la commission de leur cause et de l'examen de leurs écrits à l'archevêque, voir procès-verbal à cette date.

31. Lettre de Marca au Grand-Maître du 24 janvier 1660, ACDF, St. St. F 2a, 577.

32. Pour des raisons qui ne sont pas claires, A. Auguste (art. cit., p. 180, n. 2) qui a connaissance de la relation de Marca ne signale pas l'intervention royale.

33. Sur ce point voir D. Kostroun, art. cit. 
Carbonneau remet à Marca la censure des écrits trouvés chez les sœurs faite par les professeurs de théologie de la ville ${ }^{34}$. Les sœurs, averties de la venue de l'archevêque, désormais émissaire à la fois du monarque et du Grand-Maître, promettent de se soumettre à ce qu'il leur ordonnera. La première visite de l'archevêque a lieu le 9 novembre. Il affirme employer «trente heures de temps durant huit séances » à l'examen des religieuses les interrogeant sur deux chefs: «si dans le couvent les religieuses conféroient entre elles sur les opinions de Jansénius, et avec les estrangers en la conversation des grilles» et si certaines approuvaient ces erreurs d'une part, et d'autre part «sur la conduite qu'elles tenoient en leur Oraison mentale, et si elles la faisoient avec un esprit passif».

Certaines sœurs pensent pouvoir alors détourner la visite contre les commissaires et se plaignent de Carbonneau, inspiré selon elles par les jésuites, jaloux de ne pas se voir commettre l'entière direction du monastère. À ceci Marca répond qu'il ne connaît pas la source de la dénonciation, mais qu'elle avait certainement été encouragée par les paroles excessives des sœurs sur les questions disputées, de sorte qu'elles «doivent remercier Dieu du zèle qu'avoient eu ces dénonciateurs », puisque leur innocence était ainsi manifestée et que cela avait donné l'occasion de remettre «dans le bon chemin celles qui pourroient s'en estre éloignées ». Il loue en particulier la saisie des papiers par Carbonneau et leur examen par les théologiens.

Sur le fond, Marca se déclare satisfait des protestations de la prieure, de la sous-prieure et de la plus grande partie de la communauté, de n'avoir jamais rien senti « de contraire aux déterminations de l'Église», et de leur affirmation «qu'elles n'entendoient point les propositions condamnées de Jansénius », se contentant de savoir que le Saint-Siège les avait condamnées. Pour l'oraison de même, Marca résume ses conclusions en retenant la protestation des sœurs «qu'elles la pratiquoient suivant la méthode qui leur avoit esté enseignée au Novitiat, sans se servir d'autre façon plus subtile sous prétexte d'estre plus mystique $\gg^{35}$. Six sœurs, cependant, tout en affirmant ne pas y avoir ajouté «une entière foy à cause des condamnations du Saint-Siège», déclarent «que dans les

34. Lettre de Marca au Grand-Maître du 24 janvier 1660, ACDF, St. St. F 2a, 577.

35. Lettre de Marca au Grand-Maître du 24 janvier 1660, ACDF, St. St. F 2a, 577. 
conversations elles s'entretenoient des matières du Jansénisme», ce qui leur valut le blâme de quelques autres sœurs, et qu'elles «avoient eu connoissance de cette mauvaise doctrine tant par un Catéchisme de la Grace imprimé à Paris, que par des écrits et des entretiens familiers aux grilles $»^{36}$. Les sœurs donc auraient eu accès au Catéchisme de la Grâce du vicaire de Saint-Merry, Mathieu Feydeau, ouvrage censuré par le Saint-Office en 1650 et 1654, et dont l'auteur vit dans la clandestinité depuis son refus d'obéir à la lettre de cachet par laquelle Mazarin l'exile en $1657^{37}$.

Tant sur la doctrine de la grâce que sur celle de l'oraison, Marca leur représente la véritable doctrine d'Augustin, proposant ainsi un cours d'antijansénisme pour les simples, en citant une homélie comparant la grâce à un aliment qui fait effet "pourveu qu'on l'avale» et qui n'est d'aucun profit si on «le laisse dans la bouche», discours dont les Sœurs sont «extrêmement consolées». Marca y ajoute des preuves scripturaires et augustiniennes de l'universalité de la volonté salvifique du Christ. Quant à l'oraison passive, sur huit sœurs qui prenaient conseil du directeur qui enseignait d'attendre «avec repos d'esprit les inspirations qu'il [...] plairoit [à Dieu] de faire couler dans l'âme», Marca affirme que quatre ont déjà abandonné cette méthode en raison de la «paresse» et des «scrupules» qu'elle entraînait chez elles. L'archevêque les encourage ne pas «s'embarrasser en de nouvelles méthodes qu'elles ne pénétroient pas assez».

Marca s'entretient enfin avec la prieure et la sous-prieure et affirme sa satisfaction «de laisser leur maison entière purgée du Jansénisme». Il déclare son intention d'en faire rapport à la reine pour essayer d'obtenir de sa part une deuxième visite pour le monastère. En réponse, les sœurs réclament leurs écrits, ce que Marca conditionne à leur examen par ses soins une fois qu'il les aura reçus de Carbonneau. Elles demandent encore qu'on permette à un de «leurs directeurs séculiers» et à un Carme déchaux de continuer à les visiter. Enfin elles rappellent à l'archevêque que l'interdiction d'autres prédicateurs et confesseurs que ceux choisis par les commissaires est contraire à leurs Constitutions qui accordent

36. Ibid.

37. Mathieu Feydeau, Catéchisme de la Grâce, Paris, 1650. Sur l'auteur et le livre, voir la notice de Jean Lesaulnier, Dictionnaire de Port-Royal, col. 405-407. 
à la supérieure le choix des confesseurs extraordinaires, revenant donc encore sur la question de leurs droits communautaires. Marca défend cependant cette provision comme obligatoire dans le cadre d'une visite ordonnée par leur supérieur majeur. En réalité, Marca impose à Carbonneau l'autorisation pour la supérieure de choisir des confesseurs, mais en restreignant le recrutement aux maisons franciscaines de la ville.

À la reine, l'archevêque fait ensuite le rapport suivant: il y avait des «esprits du dehors » qui avaient travaillé à répandre dans le couvent «le venin du Jansénisme» mais la sagesse des sœurs anciennes avait su protéger les plus jeunes. Pour l'oraison mentale «la nouveauté que certains Docteurs avoient voulu introduire en la pratique, commençoit d'exciter quelque contention entre les filles», mais lui-même avait «apaisé cela en réduisant les plus curieuses à faire leurs Méditations suivant leur première et ancienne méthode». Il supplie la reine, en son nom et en celui du visiteur, d'honorer le monastère ainsi purgé de sa visite, laquelle l'accorde et s'exécute.

Dans une lettre de février 1660 au Grand-Maître, la prieure loue à son tour le deus ex machina épiscopal sans jamais reconnaître d'ailleurs un quelconque problème dans le couvent mais en célébrant sa capacité à tout mettre «a descouvert $»^{38}$. L'affaire est donc rondement menée et par la seule grâce de la visite de l'évêque et de son charisme d'enseignement voici le monastère transformé de nid de jansénistes en havre d'innocence dont tout le monde soit est satisfait (la reine, Marca), soit doit se satisfaire (le Grand-Maître, les visiteurs, et la curie romaine). À tout prendre d'ailleurs, il ne s'agit pas seulement de sauver l'intégrité des sœurs mais celle de l'archevêque lui-même puisque ce dernier avait toléré l'apostolat et l'enseignement du P. Vignaux principal mis en cause dans cette affaire.

Cependant, la douce purge épiscopale ne résout pas l'ensemble des difficultés. Elle semble même en susciter de nouvelles. À Rome, où la Congrégation du Saint-Office continue de surveiller l'affaire, on n'est guère satisfait. En septembre 1660, vraisemblablement sur ordre du pape, le nouveau Grand-Maître, Cotoner, confie la surintendance spirituelle des sœurs à l'archevêque pour une durée

38. Lettre du 14 février 1660 de la prieure au Grand-Maître, ACDF, St. St. F 2a, 579 et passim. 
d'un an $^{39}$. Il n'est pas impossible que cette commission apparaisse à Rome comme la seule forme possible d'intervention. D'un point de vue canonique par ailleurs, le cadre de la visite est maintenu. Si les commissaires en dehors de la question du temporel et des visites des confesseurs, laissent la résolution de l'affaire à Marca qui, ayant quitté Toulouse ${ }^{40}$, agit par l'intermédiaire de ses grands vicaires, ils restent le relais des ordres du Grand-Maître auprès de la communauté, notamment pour interdire aux sœurs de prendre des confesseurs autres que franciscains ou jésuites ${ }^{41}$. Avertis que la nouvelle prieure a réintroduit dans le monastère le P. Vignaux, les visiteurs se rendent auprès de ce dernier pour «le prier fort civilement de ne retourner plus là dedans ». Vignaux refuse arguant des besoins spirituels des sœurs et des demandes de la nouvelle prieure, Madame de Touges $^{42}$. Celle-ci a vraisemblablement été élue dans une réaction à la visite qui donne le pouvoir au groupe de sœurs les plus proches de Vignaux et des clercs jansénisants, groupe par ailleurs en partie structuré par des liens familiaux ${ }^{43}$. La prieure soutenant le refus des sœurs qui l'ont pour directeur de le voir exclure, les visiteurs vont alors surprendre Vignaux lors d'une de ses visites au monastère, battant «à la porte du parloir où il

39. Lettre de commission à Pierre de Marca, 3 septembre 1660, ACDF, St. St. F2a, 660, lettre à la prieure du même jour, ibid, 629, et au commissaire Carbonneau, ibid., 631: «Très Cher et bien Amé Religieux. Nostre Saint Père le Pape qui veille incessamment à la conservation des Ames qui sont dans le giron de Nre Mère la Ste Eglise aiant eu quelque information que nos Religieuses de St Jean ou quelques unes d'entr'elles n'estoient pas bien encore désabuzées des opinions que les adhérents de Jansénius avoient semé dans leurs Ames, a trouvé non seulement a propos mais entièrement nécessaire, que pour oster la dernière racine de ce mal, nous supplicassions Mons vostre Archevesque qui jusques a cette heure y a travaillé avec tant de prudence et de charité, de vouloir, durant un an, recevoir la surintendance spirituelle de nostre Couvent $[\ldots]$ ».

40. Une note dans A.D. Haute Garonne 1G 3963 rappelle son départ de Toulouse en septembre 1660, au moment donc où le Grand-Maître lui commet l'intendance spirituelle de la communauté, et que nommé à la tête du conseil de conscience en 1661 puis archevêque de Paris en février 1662, il ne revint plus dans la capitale languedocienne.

41. Procès-verbal en date du 18 mars 1661.

42. Gauside de Touges est une première fois prieure du monastère en 1654. Sa seconde élection a lieu pendant la crise qui secoue le monastère puisque l'acte de confirmation de l'élection est daté du 18 juin 1660 (voir les actes de confirmation dans A. D. Haute-Garonne 216H 34). De 1657 à 1660, la prieure est Marie de Narbonne Fimarcon. En 1663 c'est Julie de Castellane d'Alluis qui prend sa succession.

43. Voir infra. 
estoit serré par dedans tout seul avec une de ces religieuses », et lui renouvellent leur demande. À Vignaux qui se plaint qu'il peut se rendre où il veut, n'étant pas excommunié, Carbonneau répond «que chacun estoit maistre dans sa maison».

Le conflit entre les commissaires et la prieure continue de s'aggraver. Alors que ceux-ci font vœu de ne plus se mêler de la vie spirituelle de la communauté et semblent accélérer la visite du temporel pour y mettre un terme, leur procès-verbal revient sur leurs rapports avec la prieure. Elle s'obstine selon eux à ne pas se conformer à l'ordre du Grand-Maître de ne se confesser qu'aux franciscains et aux jésuites. Ils rapportent alors un entretien particulièrement violent ${ }^{44}$ :

[...] et luy dismes, vous ne vous plaindrez pas Madame de moy puisque je suis bien assez patiens vous ayant escrit et vous ne m'avez point faict response, estant venu icy, vous ne m'avez rien dict sur ce je vous avois escrit de ne revoir pas tout le monde, comme vous le faisiez, et de plus vous avez donné permission à vos Religieuses de se confesser au Recteur de Fronton et d'autres, vous sçavez que vous ne le pouvez pas. Elle nous auroit demandé de quels autre plus que du Recteur de Fronton, nous luy dismes, J'aurois honte de vous le dire car j'ay honte de v[otre] désobéissance, elle nous respondit, qu'il y avoit deux ans que nous les tyrannisions, nous luy dismes que sa vanité et désobéissance avoit mis la peste et le poison dans cette Communauté, puisqu'elle ne pensoit qu'à la commander en abusant de la bonté de la pluspart de ses religieuses, et qu'elle ne recognoissoit ny le Pape, ny le Grand Maistre, ny l'archevesque ains seulement sa seule vanité, à quoy elle nous auroit respondu diverses choses qui nous obligèrent à luy dire qu'elle estoit une impertinante, elle se leva, nous serrant le rideau du parloir où nous estions avec elle sur le nez sans nous dire adieu s'en allant en grondant contre nous, [...].

Le point de rupture est atteint. Le procès-verbal tourne au réquisitoire contre la prieure et ses sœurs, notamment la maîtresse des nocives. Il se conclut d'ailleurs sur l'exposé des raisons pour lesquelles Carbonneau se refuse à le communiquer aux sœurs: crainte qu'elles n'en abusent si on se montre par la suite indulgent et qu'elles ne se vantent d'obtenir une cassation de la visite. Peu de temps après commence la séquence polémique qui assure une nouvelle forme de publicité à l' affaire. Celle-ci est suivie d'une série d'actes publics dans lesquels les visiteurs sont encore engagés. Ainsi en juin 1661 les sœurs font enregistrer devant un notaire apostolique

44. Procès-verbal en date du 21 mars 1659. 
leur volonté, exprimée en chapitre, de faire une profession de foi en forme, incluant leur soumission à la condamnation des propositions $\mathrm{s}^{45}$. Une copie de la profession de foi est d'ailleurs envoyée à Rome pour obtenir reconnaissance de l'innocence de la communauté, mais aussi pour faire cesser l'ensemble des procédures et la visite ${ }^{46}$. Six des sœurs refusent de la signer, avec le soutien de Carbonneau.

L'archevêque, lui, semble veiller à la bonne conclusion d'une affaire dans laquelle est désormais engagée son autorité. Selon sa lettre du 29 juillet 1661, ses grands vicaires auraient présenté l'acte passé à l'initiative des sœurs comme l'équivalent de la signature du Formulaire, en même temps qu'ils obtenaient les signatures des autres communautés régulières ${ }^{47}$. De fait, la profession de foi contient une citation presque terme à terme du Formulaire ${ }^{48}$, cependant il semble que les vicaires généraux évacuent la véritable nature de l'acte. De plus, les modifications introduites dans la formule ne sont pas indifférentes. La mention du fait que l'Augustinus ne contient pas la véritable doctrine d'Augustin et que Jansenius l'a

45. ACDF, St. St. F 2b, 195. L'acte enregistre la demande du chapitre et la requête présentée aux autorités (Marca, le Grand-Maître, les grands vicaires) de recevoir une profession de foi publique, et la visite des grands vicaires en réponse à cette requête. L'acte a donc pour objet d'accompagner la profession de foi.

46. «[Votre Sainteté] est très humblement suppliée nous recevoir en sa sainte protection, nous garantir de l'oppression de nos calomniateurs, nous restablir dans la bone réputation de la foy catholique et nous maintenir dans nos usages et libre observance de nos constitutions» [nous soulignons], supplique de la prieure Gauside de Touges de la part de la Communauté au Pape, accompagnant la profession de foy, ACDF, St. St. F 2b, 200.

47. Lettre de Pierre de Marca en réponse à ses grands vicaires, reproduite par A. Auguste, art. cit., p. 186-188 [original dans le fonds des Dames Maltaises, A. D. Haute-Garonne 216H 35].

48. «Et pour faire voir que c'est avec une grande injustice qu'on soubçone qu'en nostre maison il y a de iansenistes et qui tienent les propositions condamnees de Jansenius par le Saint Siege, A ceste cause nous declarons que nous nous sommes tousiours sincerement soumise et nous soumetons a la Constitution du pape Innocent X selon son veritable sens qui a este determiné par la Constitution de Nostre Saint Pere le pape Alexandre 7. Nous avons tousiours coneu et conoissons que nous sommes obliges en conscience d'obeir a ces Constitutions. Nous avons condamne et condamnons de cœur et de bouche la doctrine des Cinq propositions dudit Cornelius Jansenius, et toute doctrine que nous nostre Saint Pere le pape condamne, protestant que nous n'avons eust et ne voulons avoir d'autre foy ni d'autre doctrine que celle de l'Eglise Romaine que nous reconnoissons pour nostre Mere dans laquelle seule est et se trouve le veritable salut, laquelle foy de l'Eglise romaine nous confessons de bon gré, looüons (sic) et prometons de tenir constamant et pourveoyerons en tant qu'il sera en nous qu'elle soit tenue et professee par toutes les Persones qui nous sont subietes », ACDF, St. St. F2b, 202. 
mal interprétée a disparu! On a là un signe certain non seulement de la connaissance des débats sur le contenu du Formulaire mais encore d'un attachement à une doctrine spécifique sur la grâce. Plus intéressant encore, les verbes exprimant la profession de foi sont redoublés au passé et, de même, la clausule initiale donne comme vocation à la signature non pas tant la profession de foi des sœurs que la publication de leur innocence.

Marca demande de substituer à cet acte la signature pure et simple du Formulaire et recommande à ses vicaires de choisir pour les sœurs un confesseur séculier, «sans aucun attachement à aucun des partis», mais qui soit cependant «ouvertement opposé au jansénisme». Pour la direction, il n'autorise l'entrée dans le couvent que de «M. de Mervilli ou de Mazas ou [du] Père Ferrier, jésuite». Il ordonne d'avertir secrètement les directeurs suspects, Vignaux, prêtre de la Mission, Le Blanc, oratorien et deux autres «de s'abstenir de hanter cette maison», sans désaveu cependant puisque Marca promet de «les employer en des fonctions plus considérables». Arnaud Baric enfin, s'il pourra venir célébrer son obit, devra s'abstenir de parler aux religieuses. À la prieure, Marca demande qu'on impose de faire une satisfaction au P. Ferrier. De même, les sœurs doivent se départir de toutes plaintes contre les docteurs qui ont fait la censure de leurs écrits.

Encore après cette lettre, le 21 août 1661, les sœurs obtiennent un acte des grands vicaires en leur faveur, mentionnant certes la visite de l'archevêque mais encore leurs propres visites et entretiens et même l'examen de leurs cellules et de leurs écrits. Ils se déclarent «pleinement satisfaicts d'elles, tant par leurs responses qui ont esté trouvées orthodoxes et catholiques; que pour n'avoir trouvé aucun livre ny escrit suspect en leur pouvoir». Sans se prononcer sur le passé, les vicaires affirment les avoir «trouvées entièrement immunes, innocentes, et nettes de toute tasche d'erreur, dogme pervers ou hérésie quelle que ce soit».

À Rome cependant la suspicion demeure entretenue par le P. Honoré Fabri auquel Jean Ferrier rappelle occasionnellement le cas toulousain, espérant encore en juin 1663 une intervention pontificale $^{49}$. Affirmant le monastère ruiné par les ecclésiastiques

49. Lettre de Fabri à Ferrier du 19 juin 1663, ACDF, St. St. F 2c, 117. Rappelons qu'à ce moment Fabri, qui est notamment l'un des traducteurs principaux des 
qui en ont pris la direction, Ferrier, dans la réponse à son confrère romain, recommande l'exclusion du gouvernement de la supérieure Gauside de Touges de Mauvoisin et des autres sœurs de sa famille ${ }^{50}$, faute de quoi «il est indubitable que ce monastère est perdu, et sera pire que le monastère de Port Royal de Paris ». Il propose aussi de transmettre tous les actes dont il dispose mais craint «qu'on ait déjà vuidé cette affaire, car il y a long tems qu'elle traisne à Rome». Il insiste en particulier sur la nécessité de soutenir les sœurs «qui ont tenu ferme dans le bon parti, et qui appartiennent à des Seigneurs de grande qualités ${ }^{51}$ lesquelles «sont fort désolées, craignant que les autres qui ont envoyé secrettement à Rome pour se faire déclarer innocentes n'ayent surpris les juges par leurs artifices [...]». En septembre 1663 cependant s'il mentionne encore les Dames Maltaises, c'est pour les présenter comme un exemple de la politique de conquête des jansénistes, à un moment où il essaie d'obtenir des restrictions aux fondations de congrégations d'inspiration janséniste dans le sud de la France et une éventuelle révocation du

documents français à l'Inquisition Romaine et en particulier pour les congrégations du jansénisme, continue de jouer un rôle fondamental au Saint-Office, malgré ses premiers échecs au moment de la censure de l'Apologie pour les Casuistes en octobre 1659. Voir J.-L. Quantin, art. cit, p. 22-23.

50. La sœur Marie de Touges est citée dans le procès-verbal de la visite comme la fondatrice de l'obit desservi par Arnaud Baric (voir ce dernier en date du 29 mai 1659). La famille de Touges est commingeoise. Jean de Touges, seigneur de Noaillan fait partie des "Quarante-cinq», la garde personnelle d'Henri III, voir Pierre-Jean Souriac, «Noblesse commingeoise et service armé du roi de France, 1560-1600», Revue de Comminges et des Pyrénées Centrales, 2005, p. 523-550.

51. Il fait ici certainement allusion aux sœurs que Rapin connaît par ailleurs, Antoinette et Françoise de Mirandol, Perside de Bénac de Navailles et la sœur de Fontenilles. Les sœurs de Mirandol sont les filles d'Étienne de Mirandol, écuyer, dont la seigneurie est située dans le vicomté de Turenne [voir Nicolas Vitton de Saint-Allais, Nobiliaire universel de France, Bachelin-Deflorenne, Paris, 1818, p. 448]. Perside de Bénac est la fille de Philippe de Montault (d. 1654), marquis de Bénac et duc de Navailles, sénéchal et gouverneur de Bigorre [voir Louis Moréri, Le Grand dictionnaire historique, vol. 6, Supplément, Paris, 1712, p. 680], F. de Fontenilles est la fille de Jean-Jacques de la Roche, baron de Fontenilles, en Comminges [Jean-Baptiste de Courcelles, Histoire généalogique et héraldique des Pairs de France, t. 1, Paris, Bertrand, Treuttel et Wurtz, 1822, p. 54]. La famille de cette dernière semble en pleine ascension sociale à ce moment. Son père comme son frère sont faits gentilshommes de la chambre du Roi. Ce dernier obtient l'érection de sa seigneurie en marquisat, avant de s'implanter en Picardie. Sans investigations plus précises, il est difficile de savoir si ces familles sont liées entre elles, et surtout si Fabri fait allusion à quoi qui ce soit d'autre qu'à leurs parents dans le passage cité. 
bref en faveur des Filles de l'Enfance ${ }^{52}$. On ne trouve guère de trace ultérieure de l'épisode qui, comme on l'a dit, disparait rapidement de la mémoire du jansénisme.

Il y a d'ailleurs de ce point de vue une distance entre l'effacement de l'affaire et l'insatisfaction générale des acteurs à l'égard d'une solution (ou d'une absence de solution) dont le véhicule est un silence que les autorités ont réussi à imposer, après une séquence polémique dans la première moitié de l'année 1661. Elle a conduit les autorités ecclésiastiques à renforcer leur intervention à laquelle la communauté semble sinon se soumettre, du moins afficher un type de soumission regardé comme suffisant par une partie des acteurs $^{53}$.

\section{Le(S) Jansénisme(s) des Maltaises}

La question de l'orthodoxie ou de l'hétérodoxie des sœurs demeure un lieu inévitable des stratégies polémiques. C'est d'ailleurs autour de la possibilité de contester l'actualité de leur hétérodoxie que s'ouvre l'espace des conflits multiformes qui s'engagent alors. Il n'est donc pas indifférent de pouvoir caractériser doctrinalement les reproches faits aux sœurs, pour comprendre justement les efforts visant à produire cette caractérisation ou à la réfuter.

À l'issue de la première visite, les commissaires font établir une «censure» détaillée des ouvrages. Elle est signée le dernier jour

52. ACDF, St. St. F 2c, 243. De même dans une lettre du P. Annat au P. Fabri de novembre 1663, le cas des Maltaises de Toulouse est présenté plus comme un exemple des mauvais effets du jansénisme que comme une affaire dans laquelle il faut encore agir, ACDF, St. St. F 2c, 300. Dans la lettre de septembre 1663, Ferrier indique par ailleurs que Vignaux bénéficie de la protection du prince de Conti, ce qui expliquerait en grande partie la liberté relative dont il jouit.

53. Et par les historiens qui croisent l'affaire. Dans le court passage qu'il consacre à l'intervention de Marca, Fr. Gaquère, son biographe, se félicite ainsi de l'efficacité de son intervention: "Ces décisions d'un administrateur avisé et charitable, remarque M. l'abbé Auguste, ramenèrent la paix dans la maison de Saint-Jean. Intransigeant sur les questions doctrinales, l'archevêque s'était montré plein de tact et de modération envers ceux et celles qui s'étaient compromis par leurs intrigues dans le Port-Royal toulousain. Ce fut pour ces religieuses un rare bonheur que de rencontrer dans Marca un juge aussi équitable qu'intelligent et d'échapper du même coup à l'intervention des grands batailleurs de PortRoyal», François Gaquère, Pierre de Marca (1594-1662). Sa vie, ses æuvres, son gallicanisme, Paris, Lethielleux, 1932, p. 258-259. 
de juillet 1659 par les frères Simplicien, et Landon, tous deux professeurs augustins, et par le frère Fraissinous, lui aussi professeur royal, mais bernardin et qui a déjà participé en Sorbonne à la censure des cinq propositions ${ }^{54}$. La censure passe ensuite entre les mains des théologiens des différents ordres qui l'approuvent: les carmes, le 31 juillet, les jésuites le 6 août et les franciscains de l'Observance le 8 . Le résultat de ce travail à la fois anonyme, sans auteur individuel identifié et à l'auctorialité duquel se substitue surtout la multiplicité des approbateurs est décalé par rapport au genre même de la censure doctrinale, telle qu'elle s'exprime dans les universités. En effet, la censure est prise dans la tension jamais résolue entre qualification doctrinale et individuelle de l'hétérodoxie ${ }^{55}$, en même temps qu'elle joue précisément de cette incertitude pour mettre en abyme ces deux modalités de production de l'hétérodoxie par un travail d'écriture bien plus que par la mise en œuvre d'une expertise de l'ars censoria si souvent posée comme caractère essentiel de l'autorité théologique ${ }^{56}$.

La censure distingue de fait quatre types d'écrits parmi ceux qui ont été transmis par les commissaires. D'abord « ceux qui sont bons et utiles aux filles Religieuses », en particulier les ouvrages de dévotion, les méthodes d'oraison et d'examen de conscience. Ensuite «ceux qui sont bons à la verité considérés en eux-mesmes, mais au regard des filles Religieuses, ils sont très impertinants et du tout inutiles », dangereux pour leur repos spirituel, car dépassant leur entendement et risquant de causer des «seicheresses» spirituelles, parce qu'ils traitent des parties les plus hautes de la théologie, en l'occurrence des questions de la Prima pars des Sommes théologiques, traitant « de la Sacrée Théologie, qu'elle est la nature de la théologie, si l'estude d'icelle est nécessaire à un Ecclesiastique, de la bonté de Dieu, [...] des mystères de la Sainte Trinité, des processions divines [...]».

De manière assez surprenante, la censure cite longuement les passages des écrits rédigés par les directeurs pour l'usage des sœurs,

54. ACDF, St. St. F 2a, 566v.

55. Voir le compte rendu de la table ronde «Fonctions de l'orthodoxie» dans Susanna Elm, Éric Rebillard, Antonella Romano (éds.), Orthodoxie, christianisme, histoire, Rome, École Française de Rome (Coll. de l'École Française de Rome, 270), 2000, p. 369.

56. Voir Bruno Neveu, L'Erreur et son juge. Remarques sur les censures doctrinales à l'époque moderne, Naples, Bibliopolis, 1993. 
« faits à perte veuë et avec des termes qui n'ont aucun sens » et qui auraient ainsi contenu des discours de ce type:

Pour arriver à la totale transfusion de la Créature en Dieu, il faut que la Créature soit perduë à son vivre, à son sentir, à son sçavoir, à son pouvoir, à son mourir, mourant sans mourir, vivant sans vivre, patissant sans patir, se résignant sans résigner, que si cela luy est inférieur à son acte électif, l'ame est alors impassible inattingible immobile, d'autant que les Créatures ne peuvent par leurs inventions atteindre à Dieu d'une infinie distance.

Et le censeur de commenter: "Qui est ce qui pourroit former quelque véritable sens de ces périodes $[\ldots]$ ? »

La troisième catégorie d'écrits identifiée par les censeurs concerne ceux qui «non seulement sont inutiles mais aussy entièrement ridicules, estant plustost des parfaits galimathias que des discours spirituels ». Là encore l'auteur multiplie les exemples tels ce passage sur la nuit des sens dans la contemplation mystique:

Dieu vous constitue et vous arreste en luy d'une manière imperceptiblement perceptible et pour l'ordinaire du tout imperceptible, tant plus cela est et se fait ainsi, tant plus et tant mieux nous sommes simples, uniques, estendus et perdus au total de nostre infiny obiect et d'une science et notion qui excède toute science et notion, de tout ce qui se puisse fecondement tirer et fluer d'icy pour exprimer et manifester cette confusion perdue et unique. Car fondant et réduisant tout en son propre abysme elle ne sçait, ne voit et ne sent rien de toute chose créée et incréée sans la différence d'elle mesme, n'estant autre que l'abysme très profond du mesme object qu'il a fait et arresté en nous ou plustost hors de nous en tout luy mesme, le plus haut estat et le plus intime est dans très simple opération de Dieu et de l'esprit par laquelle il contemple Dieu incessament en imperception par manière de dire de ce qu'il voit et contemple en arrest et stabilité ferme et immobile son divin obiect en luy et par luy mesme, duquel il est ainsy secrétement satisfait.

Cette fois, pour le censeur on a basculé dans « un jargon lequel [il] ne cognoi $[t]$ point», répétant sur des passages similaires qu'il défie «quelque subtil esprit que ce soit de trouver la clef de ce chiffre», ou évoquant un «langage de l'autre monde lequel on n'entend pas fort bien en celuy-ci». Les écrits de ce type sont au dire du censeur particulièrement abondants.

La dernière catégorie clôture cette construction par l'écriture de l'hétérodoxie formelle des moniales, en regroupant les écrits qui «contiennent une doctrine hérétique et des maximes très dangereuses à la vie spirituelle». Dans cette catégorie, le premier 
effort de l'auteur est d'abord de prouver la présence du jansénisme. Cette fois, il entre beaucoup plus dans une logique traditionnelle de qualification, citant des propositions susceptibles de se voir attribuer une note théologique précise. Et les quatre premières propositions qu'il dégage sont évidemment proches de celles par lesquelles le jansénisme a été défini :

1. St Augustin enseigne que cette grace pour son efficace avec soy, qu' elle est d'elle mesme si puissante qu'elle assuiectit à ses mouvements sans aucune résistance les cœurs les plus rebelles et obstinés que l'âme qui en est touchée et prévenu luy obéit nécessairement et amoureusement avec liberté mais sans indifférence, avec plaisir mais sans contraincte. Voilà pourquoy St Augustin l'apelle grace triomphante.

2. Sans elle il est impossible d'avoir la moindre pensée, désir, souhait (il parle de la grâce efficace).

3. La volonté agit librement toutes les fois qu'elle agit sans constraincte et qu'elle ayme quelque chose encore qu'elle l'ayme nécessairement son amour ne laisse pas d'estre libre.

4. Tout ce qu'on fait de bien à autruy sans actuele direction de l'amour et volonté de Dieu n'est que propre instinct de la Nature Ces 4 articles sont faux et parlent le pur Jansénisme.

Plus même, le censeur affirme que dans les écrits des sœurs, on a trouvé «les cinq propositions de Jansénius traduites en Français».

Enfin il s'emploie à dégager une série d'erreurs, lesquelles font plus ou moins le lien entre la doctrine mystique des sœurs et leur jansénisme avéré. Il repère ainsi l'affirmation que « la chasteté est un retranchement de tous les plaisirs et délices de la chair», mauvaise définition qui non seulement oublie que la chasteté ne renonce qu' aux plaisirs illicites, mais encore rappelle à la fois le discours mystique sur l'anéantissement trouvé dans le reste des écrits et la radicalité de la doctrine janséniste de la grâce et des bonnes œuvres. De même cette autre: «l'orgueil oste la véritable virginité aux vierges». Évoquant le conseil donné par un des directeurs selon lequel «la veüe confuse de vos defauts suffit et quand vous vous disposes à confesser, contestes vous de prendre une ou deux de vos fautes particulières mais les plus volontaires », il note immédiatement que le conseil est «trop dangereux et chocque le Concile de Trente», qui demande une confession détaillée des fautes et de leurs circonstances. Il ne manque pas de noter que le même directeur consent de s'abstenir de communier par «révérence pour JésusChrist et par l'appréhension de son ineffable pureté». C'est, écrit le censeur, «parler en Arnaudiste car si la révérence pour Jésus Christ 
et notre indignité nous doivent abstenir de la Communion, l'usage de ce sacrement sera bien tost aboly de l'Église, ce qu'Arnauld et ses collègues prétendoient». En substance, en procédant à une lecture et à une transposition générique théologienne des papiers des sœurs, il leur reproche ensuite leur manque de technicité, dans laquelle vient se loger finalement l'hétérodoxie proprement dite.

Cette appropriation n'est pas que détournement et se fonde sur l'invocation implicite d'un conflit de faculté entre théologie et mystique. Il n'est donc pas surprenant que la «doctrine spirituelle» des sœurs soit centrale dans la censure, non pas seulement pour établir leur hétérodoxie mais pour mettre en scène l'étendue de cette dernière. Les thèmes ici sont ceux de la critique théologienne de la mystique: conditions mal définies de la perfection de la prière, coopération à l'action de Dieu, passivité dans la prière, passivité dans l'effort moral. Ils sont cependant mis au service de la mise en scène du jansénisme des sœurs et de ses mauvaises conséquences. La censure absorbe des discours de registres différents et tisse entre eux des liens par son travail de taxinomie et de qualification ${ }^{57}$. De ce point de vue d'ailleurs, elle signale un état des rapports de force dans la «guerre de territoire» dont la mystique est l'objet ${ }^{58}$.

À l'évidence, un des enjeux majeurs est justement celui de l'imperméabilité de la frontière entre théologie et mystique. Le censeur le dit explicitement lorsqu'il écrit:

Quel est le dessein de ces directeurs qui donnent par escrit à des simples filles le traicté de la Grace et les divisions de la grace habituele, actuelle, prevenente, assistante, coopérante, conséquante, efficace, suffisante, première, seconde, sont elles capables de ces subtilités, ni qu'elle différence il y a entre théologie et mystique, ny de la lecture du Cantique des Cantiques duquel quelques unes ont la copie escrite à la main, et lequel il n'estoit point permis iadis de lire si ce n'est à certaines personnes agées parce qu'il ya plusieurs versets desquels pleusieurs peuvent abuser.

C'est ici la présence du langage théologique dans des écrits qui devraient se contenter de parler de vie spirituelle que le censeur réprouve. De la même manière, et à l'inverse, plusieurs des maximes

57. Sur les conflits de langage et de compétence entre théologiens, mystiques mais aussi «écrivains », voir Sophie Houdard, Les Invasions mystiques. Spiritualités, hétérodoxies et censures au début de l'époque moderne, Paris, Belles Lettres, 2008, ch. 3, «Le langage équivoque des "amateurs" de Dieu», p. 121-166.

58. Ibid., p. 166. 
posent problèmes parce que transférées dans le langage technique de la théologie elles deviennent problématiques. L'auteur de la censure relève ainsi particulièrement les maximes suivantes: «il ne faut pas estre ponctuellement en l'exercice des vertus [...], il ne faut point examiner si l'on est parfait [...] Nous nous amusons quelque fois tant à estre bons anges que nous en laissons d'estre bons hommes [...]», trois maximes dont le censeur affirme qu'elles «portent les âmes au libertinage et au relaschement de la piété et de la perfection ». Il note même qu'elles sont opposées au témoignage direct de l'Écriture (ce qui veut dire qu'il leur attribue implicitement la note d'hérésie). La censure repose évidemment sur un déplacement du lieu du discours spirituel qui lui ôte tout caractère paraclétique et toute possibilité de précision ou de correction pouvant le rendre théologiquement orthodoxe. Le problème n'est donc pas seulement pour le censeur celui de la frontière entre discours théologique et discours spirituel mais bien en creux celui de la possibilité d'une contamination de ces discours, c'est-à-dire de la porosité de cette frontière. C'est bien en réalité aussi parce que les sœurs lisent de la «théologie», qu'en retour leurs lectures spirituelles deviennent suspectes. Mais ainsi, c'est aussi l'écriture théologienne de l'hérésie des sœurs qui invente cette dernière au risque de la remise en cause à la fois de la compétence d'expertise de son auteur et de la légitimité de la saisie théologienne des discours mobilisés par la censure.

Lorsque dans la séquence polémique qui s'ouvre en 1661, la censure est mise en cause par sa publication accompagnée de Remarques nécessaires ${ }^{59}$, c'est bien cette double stratégie qui est en œuvre ce qui explique d'ailleurs peut-être paradoxalement le caractère central de la censure elle-même dans le dispositif de sa dénonciation. De fait, la censure occupe dans les Remarques le corps de l'imprimé, les «remarques» n'en constituant que l'annotation marginale, comme si la simple mise devant le public de la censure, à peu d'explications près, suffisait à en dénoncer l'illégitimité et la fragilité.

Les Remarques s'emploient donc à démontrer que le discours identifié dans les écrits des sœurs est orthodoxe en renvoyant aux sources des propositions censées constituer leur doctrine spirituelle. Une des principales autorités mobilisées dans cette entreprise de 
recharge en orthodoxie est celle de François de Sales. Cependant, les écrits des sœurs proviennent souvent d'une mystique plus spéculative. Plusieurs propositions sont ainsi attribuées au carme Jean de Saint-Samson ${ }^{60}$, mystique d'inspiration dyonisienne dont l'auteur des Remarques ne manque pas de préciser que les écrits ont été publiés à Paris et avec approbations.

En réalité, le renvoi à une mystique orthodoxe ou approuvée est insuffisant à ce moment dans l'histoire du rapport à la mystique en France ${ }^{61}$. S'y ajoute le risque supplémentaire dans ce cas, d'une véritable conjonction doctrinale entre le contenu spécifique du jansénisme et l'accusation non encore formulée comme telle de quiétisme contre le discours mystique. Cette conjonction est déjà centrale dans la dernière partie de la censure qui lie les cinq propositions à la doctrine spirituelle des sœurs. Elle est cependant dans l'esprit de plusieurs autres acteurs. Marca rapporte ainsi: «Il y en eut qui me dirent que la doctrine de la Grace efficace, qui fait agir la volonté par nécessité, quoy qu'avec plaisir, leur donnoit quelque pente à une certaine négligence de pratiquer l'oraison, présupposant que Dieu feroit tout le bien en elles sans en estre prié $»^{62}$. La Réponse au directeur inconnu ${ }^{63}$, qui critique les Remarques, reprend d'ailleurs cette mention. En réponse, l'auteur des Remarques se défend sur ce point en y opposant essentiellement le témoignage de l'assiduité des sœurs à la prière, signalant qu'une défense sur le terrain doctrinal parait alors impossible sur ce point et

60. Sur la mystique et le rôle historique de Jean de Saint-Samson, voir SuzanneMarie Bouchereaux, La Réforme des Carmes en France et Jean de Saint-Samson, Paris, Vrin, 1951, Peter-Wilhelm Janssen, Les Origines de la réforme des Carmes en France au XVII siècle, La Haye, Martinus Nijhoff, 1963, p. 233-254, et Robert Stefanotti, The Phoenix of Rennes: The Life and Poetry of John of St. Samson, 1571-1636, New-York, Berne, Frankfurt/M., Paris, Vienne, Peter Lang, 1994. Bremond en fait une figure importante de «l'invasion mystique» et s'emploie significativement à le dégager de tout soupçon de quiétisme, Histoire littéraire du sentiment religieux en France depuis la fin des Guerres de Religion jusqu'à nos jours, t. II, p. 363-393 (nouv. éd. v. 1, p. 673-693, F. Trémolières et alii éd., Grenoble, Jérôme Millon, 2006).

61. On pourrait noter par exemple que l'affaire des Dames Maltaises est exactement contemporaine de l'examen du Catéchisme de Surin à Rome et de sa dénonciation par le père Champeils. De manière tout à fait significative, c'est sur la question de l'intelligibilité du discours mystique que répond le général des jésuites Goswin Nickel, voir S. Houdard, op. cit., p. 297-298.

62. Lettre de Marca au Grand-Maître du 24 janvier 1660, ACDF, St. St. F 2a, 577.

63. Voir infra. 
à ce moment de l'histoire de la place de la mystique dans la culture confessionnelle du catholicisme français.

L'auteur des Remarques semble lui aussi avoir été conscient de cette possible conjonction, comme le montre son souci de renvoyer les propositions à des sources anti-jansénistes identifiables comme telles par le public, comme la Théologie affective de Louis Bail ${ }^{64}$. Le renvoi a alors pour fonction de rendre absurde l'accusation de jansénisme en donnant comme source des maximes des sœurs, un auteur dont l'antijansénisme est public et ancien ${ }^{65}$. De la même manière les Remarques ne manquent pas de noter de possibles sources jésuites, comme un prédicateur nommé Arnoux donné auteur de «la croix est mère et vierge aussy bien que Marie». Ainsi donc la défense de l'orthodoxie des sœurs se situe bien à un point d'intersection entre théologie et polémique. Elle joue sur la publicité des engagements polémiques des théologiens qu'elle mobilise pour désarmer le soupçon d'un jansénisme qui, en creux, n'apparaît alors plus seulement comme une réalité uniquement doctrinale.

C'est au même point d'intersection entre théologie et discours spirituel que les Remarques s'emploient à nier le caractère théologique des discours spirituels dénoncés dans la censure. La remarque $\mathrm{G}$ indique ainsi: «Les premiers traitez contiennent plus tost des matieres d'oraison que des discours de doctrine.» Ainsi l'auteur des Remarques démasque la logique de déplacement des discours mise en œuvre par la censure et souligne que «le censeur prend pour des maximes les advis particuliers qu'on donne aux âmes selon leurs dispositions et leurs besoins, et qui ne peuvent pas être communs».

À la difficulté de saisir doctrinalement l'hétérodoxie répond la volonté d'identifier cet autre point d'intersection où elle se définit en termes à la fois politiques et moraux: ceci vaut tant pour les accusateurs des sœurs que pour leurs défenseurs. C'est dans la réaction à l'accusation que les acteurs en cherchent la substance: les sœurs sont-elles inscrites dans des réseaux jansénistes? Tiennentelles publiquement des discours jansénistes? Leur attitude dénotet-elle une résistance à l'autorité typique du jansénisme? Une

64. Louis Bail, La Theologie affective ou Saint Thomas en Meditations, Paris, 1654.

65. Son De beneficio crucis dont la publication précède d'un an celle de la Théologie affective est une attaque directe contre la doctrine janséniste. 
définition politique préalable est au cœur du discernement et de la dénégation publics de l'hérésie.

C'est aussi par ce biais que la question de la grammaire de l'obéissance devient centrale: dans le procès-verbal, c'est ce que le rédacteur met en avant en retranscrivant l'échange entre le P. Ferrier et la portière du monastère. Au jésuite qui aurait indiqué qu'il venait à la requête des supérieurs légitimes, cette dernière aurait répondu : «quels supérieurs? Nous ne cognoissons point de supérieur», et le visiteur de commenter: "entendant parler de nousdit de Carboneau qui estant leur visiteur leur tenions lieu de supérieur, comme représentant de son Eminance Grand Maistre de qui nous estion déléguez $»^{66}$. Le même Carbonneau ne cesse de reprocher aux supérieures du monastère leur «impertinence» et de présenter tout appel aux respects de leurs droits comme une indiscipline. La Lettre d'un Religieux à un de ses Amys met à l'inverse en scène l'obéissance extraordinaire des sœurs, qui vont jusqu'à renoncer à leurs libertés naturelles pour se soumettre aux demandes injustes d'autorités ou iniques ou surprises, en acceptant qu'on leur dicte le choix de leurs confesseurs. L'auteur insiste même implicitement sur leur renoncement aux recours légaux ${ }^{67}$.

L'élection qui porte Gauside de Touges à la tête de la communauté semble prouver que l'enjeu, notamment autour de la question du libre choix des confesseurs, est perçu ainsi non seulement par les accusateurs et par les défenseurs des sœurs, mais aussi par les sœurs elles-mêmes. Ce n'est qu'après cette élection que le confesseur

66. Procès-verbal en date du 29 mai 1659.

67. «On leur osta du depuis la liberté de conscience que leur donne la Regle sous laquelle Elles vivent sans qu'on les ait convaincuës, ny les Confesseurs à qui Elle s'adresseoient d'aucune erreur, ou qu'on ait même pris aucune voye Canonique, et dans les formes pour les en convaincre, cét à dire qu'on les a punies de la plus rigouresues de toutes les punitions qui est la captivité de l'esprit, et la gehenne du cœur [...] Elles eussent peu sans doute, Monsieur, dans un estat si digne de compassion avoir recours au Parlement, et espérer sa protection pour estre maintenuës dans la Possession d'une liberté sous laquelle Elles ont fait leurs vœux, de laquelle Elles ont tousious iouy, et de laquelle on les a privées sans observer aucune formalité ny aucun ordre de Justice, Elles se sont neantmoins soumises à une loy si nouvelle, et si dure, sans faire sans faire seulement aucun acte de protestation pour ne pas preiudicier à leur droit. Mais quand vous sçaurez Monsieur, iusques à quel point on les a restraintes, vous admirerez encore davantage, leur humble dependance et l'amour qu'elles ont pour l'obeïssance», Lettre d'un religieux à un de ses Amys, p. 4. Nous reviendrons sur ce texte dans la deuxième partie de notre étude. 
ordinaire, le P. Bigouse, lui aussi de l'ordre de Malte, perd le soutien d'une partie de la communauté, son approbation par les deux tiers du chapitre lui étant refusée d'une voix. Surtout, la prieure fait revenir le P. Vignaux comme directeur de certaines religieuses, en sachant jouer sur les possibles conflits de juridiction entre l'archevêque et les visiteurs.

Du côté des dénonciateurs de l'hétérodoxie des sœurs, les termes de la réponse faite par Carbonneau à la prieure montrent combien l'évaluation et la qualification de la désobéissance des religieuses reposent sur une grammaire et un vocabulaire profondément informés par les relations de genre. On le voit, l'invention experte et canonique de l'hétérodoxie des Dames Maltaises repose sur la proclamation d'une série de tensions et de conflits de faculté: entre théologie et mystique, spiritualité de l'obéissance et droit, entre censure et écriture non-censurée, entre écriture autorisée et lecture sans garantie. De manière tout à fait significative, la proclamation de ces mêmes tensions et conflits de faculté est aussi au cœur de la défense de l'orthodoxie des religieuses toulousaines. Dans le jeu de miroirs de la polémique, les représentations de genre ne jouent pas un rôle indifférent; il ne s'agit pas non plus d'une simple tension supplémentaire dans le conflit que se livrent les parties prenantes de cet épisode local. Au contraire, elles se trouvent au point d'articulation de toutes ces tensions et des mobilisations contradictoires d'arguments spéculaires par les parties adverses. Il convient donc d'explorer plus avant ce lien.

(À suivre)

jpgay@unistra.fr 